\title{
Relativsätze in den Dialekten des Deutschen: Vergleich und Typologie*
}

\author{
Jürg Fleischer (Berlin/Zürich)
}

\begin{abstract}
This paper establishes a cross-dialectal typology of relative clauses in various German dialects and Yiddish according to their form and function. A great variety of different types of relativizers and relative clauses can be observed, including various pronouns, particles, and zero relatives. Combinations of these types occur, one of the most typical involving a resumptive element in a clause introduced by a particle. The Accessibility Hierarchy, a concept developed in typology, is used with great profit for this study. It turns out that for the German relativization system, a basic opposition between subject and direct object as opposed to oblique holds in virtually every variety, whereas the indirect object is much less stable. In the varieties observed, significantly more relative particles and resumptive elements occur as compared to Standard German, which turns out to be quite atypical.
\end{abstract}

\section{$1 \quad$ Einleitung}

\subsection{Theoretischer Rahmen}

Der vorliegende Beitrag befasst sich mit verschiedenen Relativsatzkonstruktionen, die in den Dialekten des Deutschen auftreten. Es wird aufgezeigt, welche Typen beobachtet werden können und in welchen grammatischen Relationen diese auftreten. ${ }^{1}$ In der allgemeinen Forschung zum Relativsatz hat sich die Accessibility Hierarchy von Keenan/Comrie (1977) als äußerst nützlich erwiesen; sie besteht aus einer Hierarchie von sechs verschiedenen grammatischen Relationen und macht Voraussagen über mögliche Systeme von Relativkonstruktionen in natürlichen Sprachen. Diese Accessibility Hierarchy sieht folgendermaßen aus: ${ }^{2}$

\footnotetext{
* Der vorliegende Beitrag ist eine leicht überarbeitete elektronische Fassung von Fleischer (2004b); die wesentlichen Ergebnisse dieses Artikels wurden im Rahmen der Tagung "Dialekte und Dialektologie an der Jahrtausendwende" (Freiburg i.Ü., 6.-7. Februar 2004) präsentiert und diskutiert. Ich danke Helen Christen und Agnès Noyer für die Organisation der Tagung und dem Publikum für die Beiträge in der Diskussion.

${ }^{1}$ In einem anderen Beitrag (Fleischer 2004a) wird aufgezeigt, wie die zu beobachtenden Konstruktionen im Rahmen der sprachtypologisch orientierten Forschung interpretiert werden können, was im vorliegenden Beitrag nur kursorisch geschehen kann. Stattdessen werden im vorliegenden Beitrag die Daten detaillierter besprochen, als dies in Fleischer (2004a) möglich ist.

${ }^{2}$ SU steht für "subject", DO für "direct object", IO für "indirect object", OBL für "major oblique case noun phrase", GEN für "'genitive' (or "possessor')" und OCOMP für "object of comparison" (Keenan/Comrie 1977: 66).
} 
$\mathrm{SU}>\mathrm{DO}>\mathrm{IO}>\mathrm{OBL}>\mathrm{GEN}>\mathrm{OCOMP}($ Keenan/Comrie 1977: 66)

Die Accessibility Hierarchy ist jenseits ihrer theoretischen Aussagekraft, auf die im vorliegenden Beitrag nicht eingegangen werden kann, ein äußerst geeignetes Mittel zur Organisation und Darstellung der Daten: viele der beobachteten Typen können nur für gewisse Positionen auf der Accessibility Hierarchy (oder nur für ein kontinuierliches Segment von Positionen) belegt werden, während in demselben System für andere Positionen andere Typen zum Zug kommen. Der vorliegende Beitrag versucht deshalb nicht nur, möglichst viele verschiedene Typen von Relativkonstruktionen aufzuzeigen, sondern fragt immer auch danach, welche Konstruktionen in welchen Dialekten für welche Positionen der Accessibility Hierarchy möglich sind; allerdings können die letzten beiden Positionen in der vorliegenden Arbeit nicht abgedeckt werden, und zwar, weil hierfür in den Quellen nicht genug Daten zur Verfügung stehen (zumindest in Bezug auf die OCOMP-Position kann auch festgehalten werden, dass sie in den untersuchten Varietäten höchstens marginalen Status hat). Ich beschränke mich also auf die vier höchsten Positionen der Accessibility Hierarchy, das heißt auf SU, DO, IO und OBL.

Im Rahmen der Dialektologie des Deutschen gibt es bisher nur eine einzige vergleichende Studie zu Relativsätzen, nämlich Weise (1917). Wie Weise (1917) bezieht auch der vorliegende Beitrag sein Material fast ausschließlich aus Dialektgrammatiken, und zwar aus folgendem Grund: Relativsätze in dialektalen Korpora sind schon an sich selten; Patocka (2000: 303) beziffert die Häufigkeit attributiver Nebensätze (von denen die Relativsätze einen Subtyp darstellen) für ein Korpus von Tonaufnahmen bairischer Dialekte in Österreich auf $8 \%$ und berichtet, dass in einer Reihe von Aufnahmen gar keine entsprechenden Beispiele vorkommen (cf. Patocka 2000: 307). Wenn nun außerdem verschiedene Systeme von Relativisierungsstrategien miteinander verglichen werden sollen, ist es essentiell, sämtliche verschiedenen Positionen der Accessibility Hierarchy möglichst lückenlos belegen zu können. Namentlich eine Position, das indirekte Objekt, ist in Korpora äußerst selten (das schließe ich zumindest aus der Auswertung von Dialekttexten); die praktisch einzige Quelle für Daten zum indirekten Objekt sind deshalb Grammatiken. Da sich herausgestellt hat, dass gerade in der IO-Position am meisten Schwankungen auftreten (cf. Abschnitt 3), ist es besonders wichtig, auch diese Position wenn immer möglich zu belegen.

\subsection{Quellen}

Die in der vorliegenden Studie berücksichtigten Daten setzen sich aus 14 verschiedenen Varietäten zusammen, die einerseits unter dem Gesichtspunkt der Vollständigkeit zusammengestellt wurden (wenn eine Grammatik nur Informationen zu einer einzigen Position der Accessibility Hierarchy enthält, ist dies für die vorliegende Arbeit keine ausreichende Basis), andererseits unter dem Gesichtspunkt einer gewissen Ausgeglichenheit verschiedener Dialektregionen. Die in Teil 2 besprochenen Typen in ihrer Gesamtheit decken zumindest einen Großteil der in deutschen Dialekten überhaupt möglichen Relativsatztypen ab. Das Korpus 
besteht aus vier niederdeutschen, drei mitteldeutschen und vier oberdeutschen Dialekten; ${ }^{3}$ außerdem werden zwei Außensprachinseln sowie das Jiddische berücksichtigt. ${ }^{4}$

Die niederdeutschen Varietäten sind drei westniederdeutsche und ein ostniederdeutscher Dialekt, und zwar zwei nordniederdeutsche, ein westfälischer und ein ostpommerscher: Die nordniederdeutsch-schleswigschen Dialekte sind vor allem durch die Ortsmundart von Husby repräsentiert (Bock 1933), einige ergänzende Daten zu den schleswigschen Dialekten stammen aus Meyer/Bichel (1983); die nordniederdeutsch-ostfriesischen Daten stehen für das Rheiderland (Wiesenhann 1936). Der westfälische Dialekt ist die in der Umgebung von Münster gesprochene Varietät (Born 1978), das Ostpommersche wird repräsentiert durch den Dialekt von Lauenburg (polnisch: Lębork), ca. 60 Kilometer westlich von Danzig.

Die mitteldeutschen Daten stehen für eine westmitteldeutsche und zwei ostmitteldeutsche Varietäten. Bei der westmitteldeutschen Varietät handelt es sich um den moselfränkischen Dialekt von Horath im Hunsrück (Reuter 1989), die beiden ostmitteldeutschen Dialekte gehören beide dem Obersächsischen an, und zwar handelt es sich um die Mundart von Leipzig (Albrecht 1881) und um den in Nordwestböhmen gesprochenen Dialekt (Hausenblas 1914).

Das Oberdeutsche wird vertreten durch einen ostfränkischen, einen nordbairischen und zwei niederalemannische Varietäten, es handelt sich um die Dialekte von Stadtsteinach (ca. 25 Kilometer nördlich von Bayreuth), des Egerlandes in Nordwestböhmen (Schiepek 1899), von Oberrotweil, ca. 20 Kilometer nordwestlich von Freiburg im Breisgau (Noth 1993), sowie von Basel (Binz 1888, Suter 1992).

Bei der einen Außensprachinsel handelt es sich um den Dialekt von Lubica (deutsch: Leibitz) in der östlichen Slowakei. Genetisch gehört dieser Dialekt zum Mittelfränkischen, weist aber auch einige ostmitteldeutsche Merkmale auf; die Sprachinsel besteht seit dem ausgehenden 12. Jahrhundert. Die andere Außensprachinsel ist das Pennsylvania-Deutsche, das von gewissen religiösen Gruppen noch heute unter anderem im amerikanischen Bundesstaat Pennsylvania (und in weiteren, teilweise benachbarten, teilweise weiter entfernten Gegenden) gesprochen wird. Genetisch steht dieser Dialekt dem Rheinfränkisch-Pfälzischen am nächsten. Die Sprachinsel besteht seit dem Ende des 17. Jahrhunderts, allerdings kamen auch nach diesem Zeitpunkt immer wieder neue Einwanderer dazu. ${ }^{5}$

\footnotetext{
3 Zur Bezeichnung der berücksichtigten Dialekte verwende ich die Klassifikation und Terminologie von Wiesinger (1983). Gewisse Dialekte bezeichne ich nur mit dem Namen des entsprechenden Dialektverbandes; dies ist in erster Linie als Hilfe zur besseren Einordnung gedacht, ich bin mir bewusst, dass beispielsweise die als "nordbairisch" oder gar nur "bairisch" bezeichneten Daten (Schiepek 1899) nicht für das gesamte Nordbairische (geschweige denn für den bairischen Dialektverband insgesamt) repräsentativ sind.

${ }^{4}$ Die Beispielsätze werden mit einer interlinearen standarddeutschen Übertragung versehen. Die in den Quellen verwendeten Transkriptionen erscheinen unverändert, in einem Fall (Reuter 1989) sind allerdings die Akzentbezeichnungen weggelassen.

5 In Fleischer (2004a) ist das Pennsylvania-Deutsche, in dem ein ansonsten kaum belegter Typ des Relativsatzes auftritt (cf. 2.6), noch nicht berücksichtigt.
} 
Etwas anders als für die deutschen Dialekte ist die Situation beim Jiddischen: hier beziehe ich mich auf die Standardsprache (und zwar in erster Linie deshalb, weil ich in Bezug auf die Dialekte nicht genügend Material finden konnte). Im Jiddischen (das im Vergleich zu anderen europäischen Sprachen spät standardisiert worden ist) ist der Unterschied zwischen den Dialekten (oder zumindest: gewissen Dialekten) und der Standardsprache sicher nicht so groß wie in anderen Fällen (tatsächlich scheinen sich viele ostjiddische Dialekte in Bezug auf die untersuchten Phänomene gleich zu verhalten wie die Standardsprache); dennoch muss im Auge behalten werden, dass die jiddischen Daten eine andere sprachliche Schicht repräsentieren als die deutschen Dialektdaten. Als jiddische Materialien dienten mir die grammatischen Beschreibungen von Mark (1978) und Jacobs et al. (1994), drei Arbeiten über den jiddischen Relativsatz (Mark 1942, Wolf 1974, Lowenstamm 1977), ein Zufallsbeleg aus einer Arbeit zur Syntax des Personalpronomens (Zaretski 1921), Beispiele aus der jiddischen Literatur sowie Materialien aus der Feldarbeit mit zwei Informantinnen. Beide leben in New York und wuchsen in jiddisch-sprachigen Familien auf (die eine ursprünglich aus Lodz, die andere aus einer Ortschaft in der Nähe von Krakau stammend). Die eine Informantin trägt regelmäßig zu einer jiddischen Wochenzeitung bei, die andere studiert jiddische Literatur; beide erteilen Jiddischunterricht und sprechen in jiddisch-sprachiger, eher formeller Umgebung für gewöhnlich Standard-Jiddisch, doch finden sich zumindest in Bezug auf die Phonologie immer wieder dialektale Züge in ihrer Sprache.

\section{Typen von Relativsätzen}

In den untersuchten Varietäten können insgesamt sieben verschiedene Marker in Relativsätzen festgestellt werden; eine primäre Unterscheidung kann zwischen flektierten und unflektierten Markern sowie durch kein overtes Element eingeleiteten Relativsätzen gemacht werden. An flektierten Markern treten die genusdifferenzierten Pronomen der, die, das und welcher, welche, welches auf, sowie die nicht genusdifferenzierten wer und was (wer und was können synchron nicht mehr unbedingt in allen Fällen als Pronomen angesehen werden; cf. 2.3 und 2.4). An Partikeln treten $d a$, wo und dass auf. Außerdem gibt es auch durch kein overtes Element eingeleitete Relativsätze, wie sie aus dem Englischen bekannt sind (vgl. the woman $\emptyset$ I love). Da bis auf $d a$ sämtliche der genannten Marker einen Relativsatz ohne irgendwelche anderen Elemente einleiten können (aber teilweise auch in Kombination mit anderen Elementen auftreten), werden diese Marker als Basis für die Typisierung in 2.1 bis 2.7 verwendet.

\subsection{Durch der, die, das eingeleitete Relativsätze}

Das Pronomen der, die, das ist in der Standardsprache die häufigste Option zur Relativsatzbildung. Dieses Relativpronomen kommt auch in gewissen Dialekten vor, Beispiel (1) illustriert dies für die DO-Position, Beispiel (2) für die IO-Position und Beispiel (3) für die OBLPosition:

dat Peerd, dat ik köfft heb

das Pferd, das ich gekauft habe (Wiesenhann 1936: 27) 
De Mann, den ik dat Geld dai

der Mann, dem ich das Geld gab (Born 1978: 61)

(3) də šēpər, von däm ęk' dåt hērd

der Schäfer, von dem ich das hörte (Pirk 1928: 27)

Von den behandelten Varietäten ist dieser Typ im Ostfriesischen für die SU- und DO-Position belegt (cf. Wiesenhann 1936: 27), im Westfälischen für alle vier Positionen (cf. Born 1978: 61-62), und im Ostpommerschen nur für die IO- und OBL-Position (cf. Pirk 1928: 2627), er tritt also in den Dialekten nicht unbedingt in derselben Distribution auf wie in der Standardsprache (in der er für alle vier Positionen Verwendung findet). Außerdem kommt dieser Typ auch im Nordbairischen und in der Mundart von Leipzig vor, allerdings in Varianz mit einem weiter unten zu besprechenden Subtyp.

Es existieren verschiedene Kombinationen des Relativpronomens der, die, das mit einem zweiten Element. Weise (1917: 70) erwähnt unter anderem einen Kombinationstyp, der allerdings in meinen Quellen nicht belegt ist, er besteht aus dem Pronomen der, die, das und einer Partikel, die dem standardsprachlichen dass entspricht. Dagegen kommen verschiedene andere Kombinationstypen in den ausgewerteten Quellen vor. Im Nordbairischen kann der, die, das mit was kombiniert werden, wie Beispiel (4) zeigt:

(4) s Hulz, ás deən wos dös g.màcht is

das Holz, aus dem was das gemacht ist (Schiepek 1899: 55)

Seiner Etymologie zufolge ist was ein (ursprünglich nur interrogativ verwendetes) Pronomen, aber es ist sehr wahrscheinlich, dass es sich in diesem Fall synchron nicht mehr um ein Pronomen handelt (cf. 2.4). Beispiel (4) zeigt diesen Typ für die OBL-Position, er kommt auch für die SU-Position vor (cf. Schiepek 1899: 55), und, zumindest nach den bei Schiepek (1899: 51) angeführten Paradigmen, auch in den dazwischen liegenden Relationen DO und IO. Wie die Angaben in Schiepek (1899) zeigen, gibt es in diesem Dialekt neben dem durch (4) repräsentierten Typ noch zwei weitere Alternativen: Neben dieser Kombination kommen auch der, die, das oder was alleine vor (im ersten Fall liegt der oben behandelte Subtyp vor, im zweiten Fall einer der in 2.4 behandelten Typen). Gemäß Schiepek (1899: 52) ist allerdings die Kombination der beiden Elemente am häufigsten.

Eine andere Kombination besteht aus dem Pronomen der, die, das und der Partikel da:

(5) der Mann, dem de das Haus gehört

der Mann, dem da das Haus gehört (Albrecht 1881: 53)

Dieser Typ ist im Nordniederdeutsch-Schleswigschen und in der obersächsischen Varietät von Leipzig belegt, und zwar im Schleswigschen nur für die SU-Position (cf. Bock 1933: 104), in Leipzig dagegen für die SU-, DO- und IO-Position (cf. Albrecht 1881: 53). Beispiel (5) zeigt diesen Typ für die IO-Position (gemäß den Angaben von Albrecht 1881: 53 alterniert dieser Typ mit einfachem der, die, das; die Kombination ist sehr häufig für die SUPosition, für die DO- und IO-Position ist dagegen einfaches der, die, das üblicher). 
Ein weiterer Kombinationstyp besteht aus dem Pronomen der, die, das und der Partikel wo, dieser Subtyp ist im Moselfränkischen und im Ostfränkischen belegt, wie die Beispiele (6)-(8) zeigen:

(6) de: $\operatorname{man}(d \varepsilon:)$ vo: if gəzein hon

der Mann, (den) wo ich gesehen habe (Reuter 1989: 266)

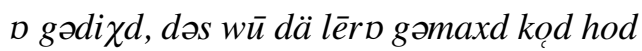

ein Gedicht, das wo der Lehrer gemacht gehabt hat (Schübel 1955: 332)

(8) di: lait denə vo: if dat gerlt gen hon

die Leute, denen wo ich das Geld gegeben habe (Reuter 1989: 266)

Für das Moselfränkische enthält Reuter (1989: 266) Belege für die obersten drei Positionen, für das Ostfränkische sind bei Schübel (1955: 332) alle vier Positionen belegt. Im Moselfränkischen ist das Pronomen für die höheren beiden Positionen fakultativ, wie (6) für die DOPosition zeigt (falls es weggelassen wird, liegt ein anderer Typ vor, cf. 2.5), für die IOPosition hingegen obligatorisch (cf. (8)); im Ostfränkischen hingegen scheint das Pronomen auch für die höheren Positionen üblich zu sein, obwohl dort auch wo alleine vorkommt (cf. Schübel 1955: 332-333).

\subsection{Durch welcher, welche, welches eingeleitete Relativsätze}

Das Pronomen welcher, welche, welches ist neben der, die, das die andere grundsätzliche Option zur Relativsatzbildung in der Standardsprache. Wie der, die, das flektiert es nach Kasus, Numerus und Genus. Von den untersuchten Varietäten kommt dieses Pronomen nur im Westfälischen und Jiddischen vor (und zwar immer nur allein, es gibt keine Kombinationen mit Partikeln; generell zeigt dieses Pronomen dasselbe Verhalten wie in der neuhochdeutschen Standardsprache. Ich vermute, dass es sich in den beiden Varietäten, in denen es vorkommt, um einen eher künstlichen Typ handelt): ${ }^{6}$

\footnotetext{
6 Der Typ welcher, welche, welches, der übrigens vergleichsweise jung ist, scheint in den europäischen Sprachen generell eher eine schriftsprachlich induzierte Entwicklung zu sein (cf. Fleischer 2004a: 236). In Bezug auf das Westfälische kann bemerkt werden, dass es sich bei diesen Daten (Born 1978) um eine der jüngsten Quellen handelt; eine stichprobenartige Durchsicht älterer westfälischer Materialien ergab keinerlei Hinweise auf diesen Typ. In Bezug auf das Jiddische müssen wir uns hier daran erinnern, dass die Daten eine Standardsprache repräsentieren, nicht Dialekte (cf. 1.2). Meine Informantinnen akzeptieren beide velkher als Relativpronomen; für die eine Informantin wäre ein durch vos eingeleiteter Relativsatz die natürlichere Variante, während die andere das genaue Gegenteil aussagt. Wahrscheinlich ist es kein Zufall, dass die Informantin, die eher velkher verwenden würde, diejenige ist, die regelmäßig Zeitungsartikel schreibt: in einem Aufsatz aus den 1920er Jahren heißt es diesbezüglich: "Der Anschluss mit vos ist volkstümlicher als mit velkher; es schadet nicht, wenn man dies für unsere Zeitungssprache im Sinn behält. [der farbund mit "vos" iz folkstimlekher, vi mit "velkher", s'shodt nit, m'zol dos in zinen hobn in undzer tsaytung-shprakh.]" (Zaretski 1921: 10). Wahrscheinlich ist das Relativpronomen velkher im Jiddischen schlussendlich eine Entlehnung: die deutsche Standardsprache, in der dieser Typ ebenfalls vorkommt, hatte teilweise einen ziemlich starken Einfluss auf die jiddische Standardsprache im 19. und beginnenden 20. Jahrhundert (cf. Weinreich 1973, 2: 74; Weinreich 1980: 418-419). Außerdem könnte es eine Rolle spielen, dass im Russischen ein sehr ähnlicher Typ vorkommt: Das Russische ist keine der primären slavischen Kontaktsprachen des Jiddischen, war aber die offizielle Sprache im Zarenreich (zu dem der zahlenmäßig mit Abstand größte Anteil der Jiddisch-Sprecher gehörte). Auch das Russische hatte deshalb einen
} 
Dat Teeken, well he maolt harr

das Zeichen, welches er gemalt hatte (Born 1978: 61)

(10) a fraynd, velkhn ikh hob shoyn etlekhe yor nit gezen

ein Freund, welchen ich habe schon mehrere Jahre nicht gesehen (Mark 1942: 130)

Beispiele (9) und (10) zeigen diese Konstruktion für die DO-Position. Im Jiddischen ist dieser Typ für alle vier Positionen der Accessibility Hierarchy möglich (cf. Lowenstamm 1977: 199; Mark 1942: 130; Mark 1978: 245), im Westfälischen ist er dagegen nur für die obersten drei belegt (cf. Born 1978: 61-62).

\subsection{Durch wer eingeleitete Relativsätze}

Das Pronomen wer zur Einleitung eines Relativsatzes ist nur im Westfälischen und Jiddischen belegt. Allerdings bestehen wesentliche Unterschiede in Bezug auf das syntaktische Verhalten dieses Elementes in den beiden Varietäten. Im Jiddischen ist dieser Typ für die SU-Position unmöglich, aber in den restlichen drei Positionen belegt, Beispiel (11) zeigt diesen Typ für die IO-Position (Belege für die DO- und OBL-Position finden sich beispielsweise bei Lowenstamm 1977: 199). Im Jiddischen ist die Form vemen eindeutig flektiert und kommt nur vor, falls das relativisierte Nomen auf Menschen referiert (wie das ja in analoger Weise auch für das standarddeutsche Interrogativpronomen wer gilt).

(11) di froy vemen er hot gegebn dos bukh

die Frau, wem er hat gegeben das Buch (Jiddisch; Informantinnen)

Im Westfälischen ist die Form we für die SU-Position belegt (cf. Born 1978: 61), sowie für die DO- und IO-Position, wie die Beispiele (12) und (13) zeigen:

(12) De Saot, we ik saiet häff

die Saat, wer ich gesät habe (Born 1978: 61)

(13) De Fru, we he 'n Teeken gaff

die Frau, wer er ein Zeichen gab (Born 1978: 61)

Die Form we zeigt keine Kasusflexion, und zwar in keiner der belegten Positionen; man beachte, dass das Relativpronomen der, das diachron gesehen über dasselbe Affix verfügt, in diesem Dialekt durchaus über flektierte Formen verfügt, cf. Beispiel (2). Außerdem kann, wie Beispiel (12) zeigt, we auch verwendet werden, wenn das relativisierte Nomen nicht auf Menschen referiert (dies ist weder beim standardsprachlichen Interrogativpronomen wer noch beim jiddischen Relativpronomen der Fall); das westfälische we hat also einen ganz anderen Status als das jiddische vemen.

Einfluss auf gewisse Register des Jiddischen (cf. Weinreich 1973, 2: 253; Weinreich 1980: 591). Das russische Pronomen kotoryj kann als Relativpronomen verwendet werden, in genau derselben Weise wie das jiddische velkher oder das standarddeutsche welcher. - Auch gemäß Wolf (1974: 42-43) handelt es sich bei velkh als Relativpronomen um eine Entlehnung aus dem Deutschen, allerdings wird eine etwas andere Argumentation verwendet. 


\subsection{Durch was eingeleitete Relativsätze}

Ziemlich häufig begegnet in den untersuchten Varietäten relativsatzeinleitendes was. Wie bei wer, das ebenfalls auf ein Interrogativpronomen zurückgeht, muss allerdings bei was zwischen verschiedenen Subtypen unterschieden werden. In gewissen Dialekten ist es so, dass die Form was das neutrale Relativpronomen das ersetzt. Für das Nordniederdeutsch-Ostfriesische des Rheiderlandes kommt nach den Angaben von Wiesenhann (1936: 27) sowohl das als auch was vor. Man beachte, dass das gegebene Beispiel, hier als (14) wiedergegeben, bis auf das relativsatzeinleitende wat exakt Beispiel (1) entspricht, wo statt der dialektalen Entsprechung von was die dialektale Entsprechung von das verwendet wird.

(14) dat Peerd, wat ik köfft heb

das Pferd, was ich gekauft habe (Wiesenhann 1936: 27)

(15) en Stück, wat druckt wät

ein Stück, was gedruckt wird (Born 1978: 61)

Dieser Typus kommt neben dem Nordniederdeutsch-Ostfriesischen auch im Westfälischen und im Obersächsischen von Leipzig vor (cf. Wiesenhann 1936: 27, Born 1978: 61, Albrecht 1881: 53), und zwar sowohl in der SU- als auch in der DO-Position (nur im Obersächsischen von Leipzig sind allerdings beide Positionen belegt). In Bezug auf die IO- und OBL-Position verfüge ich über keine Informationen; da das Interrogativpronomen was, aus dem das relativsatzeinleitende was hervorgegangen ist, über keine flektierte Dativform (mehr) verfügt, denke ich nicht, dass was in diesen Positionen auftreten kann.

Ein Unterschied zwischen dem Westfälischen einerseits und dem Ostfriesischen und Obersächsischen andererseits besteht darin, dass in letzteren sowohl das als auch was im Neutrum verwendet werden kann (cf. Wiesenhann 1936: 27, Albrecht 1881: 53), wogegen im Westfälischen das scheinbar vollständig durch was ersetzt worden ist (jedenfalls findet sich kein entsprechendes Beispiel, und auch im Paradigma des Relativpronomens, das Born 1978: 61 ansetzt, findet sich nur die Form wat). Im Westfälischen scheint also bereits ein suppletives Paradigma entstanden zu sein, während in den anderen Dialekten noch Varianz zwischen das und was besteht. Im obersächsischen Dialekt von Leipzig kommt außerdem, wie Beispiel (16) zeigt, auch bei was die Kombination mit $d a$ vor, was ganz parallel zu den Formen des Pronomens der, die, das ist (cf. 2.1); auch was ohne da ist für die SU- und DO-Position belegt (cf. Albrecht 1881: 53).

(16) das Thier, was de da rennt

das Tier, was da da rennt (Albrecht 1881: 53)

Neben was, das mit Formen des Pronomens der, die, das interagiert, findet sich auch ein anderer was-Typ in den untersuchten Varietäten, welcher kaum mehr als Pronomen zu analysieren ist; er ist in den folgenden Beispielen illustriert:

(17) də mån, wåt då $r$ wēr

der Mann, was da war (Pirk 1928: 26-27)

(18) s ros vozər frkǽuft hat

das Pferd, was er verkauft hat (Lumtzer 1896: 515) 
(19) on Menschn, wos nemmats on Pf[ennich] gitt

einem Menschen, was niemand einen Pfennig gibt (Schiepek 1899: 52)

(20) dos ferd, af vos er zitst

das Pferd, auf was er sitzt (Wolf 1974: 38)

In zwei Grammatiken wird explizit darauf hingewiesen, dass dieses was nicht flektiert ist (Hausenblas 1914: 102, Schiepek 1899: 52). In dieser Verwendung kann was auch dann auftreten, wenn das vorangehende Nomen nicht neutrales Genus aufweist (wie das beim oben diskutierten Subtypus von was der Fall gewesen ist; Beispiele (17) und (19) weisen ein Nomen auf, das nicht neutral ist). Es kann auch nicht nur dann auftreten, wenn das relativisierte Nomen nicht auf Menschen referiert, wie das beim Pronomen was der Fall ist (Beispiele (17) und (19) weisen Nomen auf, die auf Menschen referieren). Diese Charakteristika zeigen, dass es sich hierbei trotz der oberflächlichen Identität des den Relativsatz einleitenden Elementes um einen fundamental anderen Typus handelt; zumindest für gewisse Varietäten ist es berechtigt, dieses was synchron als Partikel zu analysieren (cf. Abschnitt 3).

Dieser Typus ist im Nordbairischen belegt, und zwar gemäß den Angaben von Schiepek (1899: 54-55) für alle vier Positionen (allerdings stellt die Kombination mit der, die, das in diesem Dialekt eine Alternative dar; cf. 2.1), er kommt außerdem vor im Obersächsischen Nordwestböhmens (Hausenblas 1914: 102 enthält Belege für die SU-, DO- und IO-Position), im Ostpommerschen (Pirk 1928 enthält bloß einen Satzbeleg für die SU-Position, gemäß dem Paradigma in Pirk 1928: 26 tritt dieser Typ aber auch in der DO-Position auf), in der Sprachinsel Lubica (wo er nur für die DO-Position belegt ist) und im Jiddischen (wo er nur in den beiden höchsten Positionen möglich ist, vgl. unten). Beleg (17) zeigt die SU-Position, (18) die DO-Position, (19) die IO-Position, ${ }^{7}$ und (20) die OBL-Position (für ein paralleles bairisches Beispiel cf. Schiepek 1899: 55). ${ }^{8}$

\footnotetext{
${ }^{7}$ In der IO-Position ist diese Konstruktion nur für das Nordbairische und das Obersächsische Nordwestböhmens belegt; für das Jiddische wissen wir explizit, dass diese Struktur in dieser Position unmöglich ist (cf. Beispiel (23)). Auch in den beiden Dialekten, in denen diese Konstruktion in der IO-Position auftritt, scheinen Restriktionen aufzutreten: Gemäß Schiepek (1899: 52) ist sie nur möglich, wenn das vorangehende Nomen im Dativ steht (dies könnte auch beim einzigen obersächischen Beispiel bei Hausenblas 1914: 102 der Fall sein, doch werden dort keine diesbezüglichen Restriktionen erwähnt).

${ }^{8}$ Gemäß Wolf (1974: 38) ist dieser Typ im Jiddischen nur möglich, wenn das relativisierte Nomen nicht auf eine Person referiert, wie das in Beispiel (20) der Fall ist, nicht aber in Beispiel (i). Dazu merke ich allerdings an, dass meine Informantinnen den Beispiel (20) entsprechenden, nach Beispiel (26) geformten Satz *der instrument, mit vos er hot gearbet ziemlich eindeutig ablehnen. Dies ist allerdings kein Grund, gänzlich zu bezweifeln, dass dieser spezielle Subtypus auftreten kann: neben dem bei Wolf (1974) gegebenen Beispiel kommt er ja gemäß Schiepek (1899: 55) auch im Nordbairischen vor. Die Tatsache, dass dieser Typ existiert, stellt ein Problem für die Analyse dar (das mutatis mutandis auch für das Bairische gilt): Für vos, wie es in den Beispielen (22), (23) und (26) vorliegt, ist die Annahme am plausibelsten, dass es sich um eine Partikel handelt, in (i) hingegen scheint es sich eher um ein Pronomen zu handeln, jedenfalls ist das Auftreten nach einer Präposition ein starker Hinweis zugunsten dieser Analyse. Umgekehrt kann vos im Jiddischen auch eindeutig zur Einleitung eines nicht-relativen Nebensatzes verwendet werden, wie (ii) zeigt. Vorderhand kann deshalb nur festgehalten werden, dass vos im Jiddischen scheinbar noch Reste pronominalen Verhaltens zeigt, aber in vielen Fällen eher als Partikel zu analysieren ist.
} 
Unflektiertes was kommt, wie wir gesehen haben, in vielen Varietäten nur für die höheren Positionen der Accessibility Hierarchy vor; für tiefere Positionen wird dagegen in gewissen Varietäten eine andere, nahe verwandte Konstruktion verwendet, nämlich unflektiertes was in Kombination mit einem Personalpronomen. Dieser Typus kommt in der Sprachinsel Lubica und im Jiddischen vor, und zwar ist er in Lubica für die ersten drei Relationen belegt (cf. Lumtzer 1896: 514-515) und im Jiddischen für alle vier (die jiddischen Beispiele (22) und (23) illustrieren die SU- und IO-Position, cf. z.B. Mark 1942: 130 für ein Beispiel zur DOPosition und Jacobs et al. 1994: 416 für ein Beispiel zur OBL-Position). Wie die Beispiele (21)-(23) zeigen, ist dieser Typ auch für die SU- und DO-Position möglich (bzw. scheint sogar üblicher zu sein; meine jiddischen Informantinnen bevorzugen diese Konstruktion statt des oben vorgestellten was ohne Pronomen, für Lubica fehlen entsprechende Informationen), aber in den tieferen Positionen scheint dies die einzig mögliche Konstruktion zu sein: für das Jiddische zeigen dies die Beispiele (22) und (23) mit expliziten Grammatikalitätsurteilen (die meine Informantinnen bestätigen).

(21) dr maorərméystr, vọsər bae uns hat gəọrpt

der Maurermeister, was_er bei uns hat gearbeitet (Lumtzer 1896: 514)

(22) a yidene, vos zi/Ø hot geheysn Yente

eine Jüdin, was sie/ $\emptyset$ hat geheißen Yente (Jacobs et al. 1994: 416)

(23) a melamed, vos es iz im/*? Ø zeyer shlekht gegangen

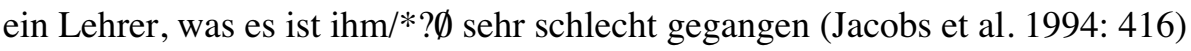

Ein weiterer Subtyp kombiniert unflektiertes was mit der Partikel $d a$ (dass dies bei flektiertem was vorkommt, ist oben bereits dargelegt worden). Für diesen Subtyp konnte ich nur ein einziges Beispiel aus dem Nordniederdeutsch-Schleswigschen finden. In Beispiel (24) bezieht sich was auf ein (pluralisches, nicht neutrales) Nomen, das auf Menschen referiert, was bei flektiertem was unmöglich wäre:

(24) Alle Mannslüd wat dor sind ...

alle Männer, was da sind ... (Bock 1933: 104)

Zwei sehr seltene Subtypen zeigen unflektiertes was in Verbindung mit einer Präposition (die allenfalls um ein zusätzliches Element erweitert ist). Diese Subtypen konnte ich einerseits im Nordniederdeutsch-Schleswigschen feststellen (in diesem Dialekt steht eine bloße Präposition allein im Nebensatz, wie in Beispiel (25)), andererseits im Jiddischen (im Jiddischen ist die Präposition um ein $d a$ erweitert, sie bildet also ein Pronominaladverb). ${ }^{9}$ In beiden Beispielen ist das vorangehende Nomen zwar unbelebt (im Jiddischen sind Pronominaladverbien wie im Standarddeutschen auf unbelebte Referenten eingeschränkt), aber sie weisen nicht neutrales

(i) $\quad$ ?* di froy, af vos er farlozt zikh

die Frau, auf VOS er verlässt sich (Wolf 1974: 38)

(ii) $\quad s^{\prime}$ iz efsher an aveyre, vos mir lakhn azoy fun a fremdn mentshn

es ist vielleicht eine Sünde, VOS wir lachen so über einen fremden Menschen (Literatur).

${ }^{9}$ Die Konstruktion mit einem Pronominaladverb, wie sie durch (26) repräsentiert wird, scheint eher marginal zu sein: meine Informantinnen würden stattdessen den sehr ähnlichen Subtypus, der ein Personalpronomen aufweist und durch Beispiel (23) repräsentiert ist, klar bevorzugen. 
Genus auf, was zeigt, dass das was in diesen beiden Beispielen nicht als Pronomen zu analysieren ist.

(25) dad is no dulə tīd vad vī lęm in

das ist eine schlechte Zeit, was wir leben in (Bock 1933: 104)

(26) der instrument vos er hot gearbet dermit

das Instrument, was er hat gearbeitet damit (Jiddisch; Informantinnen)

\subsection{Durch wo eingeleitete Relativsätze}

Bereits bei der Besprechung des Kombinationstyps der, die, das + wo (cf. 2.1) hat sich gezeigt, dass im Moselfränkischen (und Ostfränkischen) statt dieser Kombination auch einfaches wo (allerdings nur in den höheren Positionen) auftreten kann. Dieser Typus, und zwar scheinbar als einzige Möglichkeit zur Relativsatzbildung, kommt auch in den beiden niederalemannischen Dialekten vor, und zwar in Basel nur für die beiden höheren Positionen, in Oberrotweil dagegen auch für die IO-Position, wie Beispiel (29) zeigt (cf. Noth 1993: 418 zur SU- und IO-Position):

(27) Das isch e Fisch wò fliegt das ist ein Fisch, wo fliegt (Suter 1992: 165)

(28) Dä Ma, won i gestert gse ha der Mann, wo ich gestern gesehen habe (Binz 1888: 61)

(29) Mr hán scho zwe soonigi, wu-mr nid därf gläubá wir haben schon zwei solche, wo man nicht darf glauben (Noth 1993: 419)

Ein nahe verwandter Subtyp, der mit einfachem wo interagiert, besteht aus einem durch wo eingeleiteten Relativsatz, in dem die syntaktische Rolle des relativisierten Nomens im Relativsatz durch ein Personalpronomen enkodiert wird (diese Konstruktion ist parallel zum in der Sprachinsel Lubica und im Jiddischen auftretenden Subtyp was + Personalpronomen, mit dem einzigen Unterschied, dass dort der Nebensatz durch was statt wo eingeleitet wird; cf. 2.4). Dieser Subtyp kommt im niederalemannischen Dialekt von Basel für die IO-Position vor, wie Beispiel (30) zeigt, und scheint für diese Position die einzige Möglichkeit zu sein; im niederalemannischen Dialekt von Oberrrotweil dagegen scheint dieser Subtypus im IO unbekannt zu sein, stattdessen wird bloßes wo verwendet (cf. Beispiel (29)). In beiden niederalemannischen Dialekten ist dieser Typus dagegen für die OBL-Position belegt (cf. Noth 1993: 419 für ein analoges Beispiel zu (31)).

(30) Dä Ma, woni im s Mässer gä ha dieser Mann, wo ich ihm das Messer gegeben habe (Binz 1888: 61)

(31) die Lyt wo mer iber si gschwätzt händ die Leute, wo wir über sie geschwatzt haben (Suter 1992: 165)

Zwei weitere Subtypen bestehen aus einem durch wo eingeleiteten Relativsatz, in dem später eine bloße oder durch $d a$ erweiterte (also ein Pronominaladverb bildende) Präposition auftritt, wie dies in den Beispielen (32) und (33) der Fall ist: 
en ... Fro, wo ik heel groten Respekt vor heff

eine ... Frau, wo ich unmäßig großen Respekt vor habe (Meyer/Bichel 1983: 169)

(33) ə onərs gfēs, wū mər draus asn ōwer dringy sel

ein anderes Gefäß, wo man draus essen oder trinken soll (Hausenblas 1914: 138)

Diese Subtypen, die offensichtlich auf OBL beschränkt sind, sind in den deutschen Dialekten sehr weit verbreitet: die Konstruktion ohne $d a$ kommt in praktisch sämtlichen niederdeutschen Dialekten vor, der Subtypus mit $d a$ stellt das hochdeutsche Analogon zu dieser Konstruktion dar (cf. Fleischer 2002: 190-191 bzw. 262-264 mit den Karten 3 und 6). Im Standarddeutschen können sich Pronominaladverbien nicht auf menschliche Referenten beziehen; unter den Dialekten gibt es solche, für die diese Restriktion gilt, und solche, in denen dies nicht der Fall ist, wie Beispiel (32) zeigt. In Bezug auf diese beiden Konstruktionen, die (in der oben beschriebenen Distribution) praktisch im gesamten binnendeutschen Sprachgebiet vorkommen (interessanterweise allerdings weder in der Sprachinsel Lubica noch im Jiddischen), zeigt sich ein fundamentaler Unterschied zwischen den Relativisierungsstrategien der Standardsprache (in der für diese Position bei unbelebten Nomen nur ungetrennte Pronominaladverbien möglich sind) in Opposition zu praktisch sämtlichen Dialekten; interessanterweise kommen ungetrennte relativische Pronominaladverbien in den Dialekten des Deutschen praktisch gar nicht vor (cf. Fleischer 2002: 121).

Ein weiterer, seltenerer, aber nahe verwandter Subtypus wird schließlich durch Beispiel (34) repräsentiert: hier sind im Nebensatz zwei $d a$ präsent, einmal unmittelbar vor der Präposition, einmal nach dem nebensatzeinleitenden wo. Diesen Subtypus habe ich nur in der Mundart von Leipzig feststellen können, wo in höheren Positionen ebenfalls Kombinationen auftreten, deren zweites Element $d a$ ist (cf. 2.1, Beispiel (5), 2.4, Beispiel (16)).

(34) das eenzge Haus ..., wo de keene Kneipe drinne is

das einzige Haus ..., wo da keine Kneipe drin ist (Albrecht 1881: 53)

\subsection{Durch dass eingeleitete Relativsätze}

Nach Weise (1917: 71) können in gewissen ostfränkischen und bairischen Dialekten in seltenen Fällen Relativsätze mit einer Partikel eingeleitet werden, die standardsprachlichem dass entspricht; zu diesem Typ habe ich aus dem binnendeutschen Gebiet keine Quelle finden können, die genügend Information enthält, er kommt jedoch im Pennsylvania-Deutschen vor. Neben der Partikel wu, die dem standardsprachlichen wo entspricht (cf. 2.5), kann in dieser Sprachinsel ein Relativsatz auch mit der Partikel as ${ }^{10}$ eingeleitet werden, wie Beispiel (35)

10 Die Form ass ist die reguläre Entsprechung sowohl des neutralen Pronomens das als auch der (diachron letztlich ebenfalls aus dem Demonstrativpronomen hervorgegangenen und von letzterem nur graphisch geschiedenen) subordinierenden Konjunktion dass, wie Beleg (i) zeigt. Außerdem ist ass auch die Entsprechung zu als, wie Beispiel (ii) zeigt. Allerdings halte ich es für ziemlich eindeutig, dass das relative ass mit der Konjunktion, die neuhochdeutsch als als erscheint, nichts zu tun hat: In den binnendeutschen Mundarten ist scheinbar nirgends als zu einem Relativmarker geworden (jedenfalls konnte ich keine entsprechenden Hinweise finden) und die natürlichste Erklärung für den ass-Typ im Pennsylvania-Deutschen - neben der Möglichkeit, dass ein altes Muster vorliegt (wie bereits erwähnt kommt dieser Typ selten auch in den binnendeutschen Dialekten vor, cf. 
für die SU-Position zeigt (cf. Haag 1982: 225 für ein analoges Beispiel zur DO-Position). In der OBL-Position tritt das Element ass in Verbindung mit einem Pronominaladverb auf (was in analoger Weise auch bei was und wo vorkommt, cf. 2.4, 2.5), wie (36) zeigt:

(35) der Mann, ass der Schtorkipper is

der Mann, dass der Ladenbesitzer ist (Haag 1982: 225)

(36) es Kaendi ..., ass er geguckt hot defor

das Bonbon ..., dass er geschaut hat dafür (cf. "the candy that he was looking for") (Haag 1982: 226)

\subsection{Durch $\emptyset$ eingeleitete Relativsätze}

Der letzte Typ, den ich in meinem Korpus habe feststellen können, ist in deutschen Varietäten sehr selten, es handelt sich um durch kein overtes Element eingeleitete Relativsätze. Diesen Typ konnte ich nur im Nordniederdeutsch-Schleswigschen feststellen, er wird durch Beispiel (37) demonstriert. Hier muss man sich fragen, ob überhaupt ein Nebensatz vorliegt: an sich würde man erwarten, dass in diesem Fall das finite Verb am Ende steht. In diesem Dialekt jedoch (der zahlreiche dänische Merkmale aufweist) kann in einem Nebensatz das flektierte Verb unmittelbar nach dem Subjekt stehen, allfällige weitere Konstituenten können dann dem flektierten Verb folgen (cf. Bock 1933: 107 in Bezug auf dass-Sätze und Bock 1933: 104 in Bezug auf durch overte Elemente eingeleitete Relativsätze). Da der zweite Teilsatz in (37) das Nomen Rägen 'Regen' näher bestimmt und allein kaum einen grammatischen Satz darstellt, kann (37) also, wie dies Bock (1933) tut, durchaus als Relativsatz angesprochen werden. Beispiel (37) ist das einzige Beispiel für diesen Typ des Null-Relativsatzes, das ich finden konnte; es zeigt die DO-Relation, für andere Positionen, insbesondere für die SU-Position, konnte ich keine Belege finden.

(37) de schöne, warme Rägen, Øwi harren vörgestern

der schöne, warme Regen, $\emptyset$ wir hatten vorgestern (Bock 1933: 104, $\emptyset$ hinzugefügt)

Hingegen existiert noch ein weiterer Subtyp des Null-Relativsatzes, der durch Beispiel (38) repräsentiert wird:

(38) Ween dor weer Een, $\emptyset$ dor nich wull

wenn da war einer, $\emptyset$ da nicht wollte (Bock 1933: 104; $\emptyset$ hinzugefügt)

Dieser Subtyp sollte als aus einer Kombination von $\emptyset$ und $d a$ bestehend analysiert werden . Eine alternative Analyse würde darin bestehen, einen eigenen Typ des da-Relativsatzes anzusetzen, das heißt, dass bei (38) der Relativsatz nur durch $d a$ allein eingeleitet würde. Doch weil in diesem Dialekt auch Kombinationen von anderen Elementen mit $d a$ vorkommen (cf. 2.1 und 2.4), und weil die Partikel $d a$ in den übrigen Materialien immer nur in Kombina-

Weise 1917: 71) - ist wohl darin zu sehen, dass das Muster der englischen that-Relativsätze übernommen worden ist.

(i) Es iss net oft bassiert, ass ich allee ins Schteddel bin es ist nicht oft passiert, ASS ich allein ins Städtlein bin (Haag 1982: 214)

(ii) Sei Keit fliegt heecher ass meini sein Drachen fliegt höher ASS meiner (Haag 1982: 106) 
tion mit einem weiteren Element, jedoch nie allein, vorkommt, halte ich die vorgeschlagene Analyse für adäquat; immerhin kommt im selben Dialekt auch ein anderer Subtyp des NullRelativsatzes vor, wie Beispiel (37) zeigt. Es ist vielleicht kein Zufall, dass dieser Subtyp für die SU-Position belegt ist, wogegen für den eigentlichen Null-Relativsatz keine Beispiele für die SU-Position gefunden werden konnten (cf. Fleischer 2004a: 226).

\section{Schlussfolgerungen}

In diesen Schlussfolgerungen ziehe ich einige Verallgemeinerungen, die aus den in Abschnitt 2 vorgestellten Daten und Distributionen von Typen gezogen werden können (die hier kurz vorgestellten Schlüsse sind in Fleischer (2004a) detaillierter ausgeführt).

In Bezug auf die Accessibility Hierarchy kann festgestellt werden, dass in beinahe jedem Dialekt eine grundsätzliche Opposition zwischen SU und DO einerseits und OBL andererseits besteht, d.h. für die SU- und DO-Position werden dieselben Strategien verwendet, für die OBL-Position dagegen eine andere (am häufigsten belegt sind hier die in 2.5 vorgestellten Kombinationen von wo $(+d a)+$ Präposition). Die Position dazwischen, IO, geht manchmal mit SU/DO, manchmal mit OBL. Besonders interessant ist diesbezüglich, dass sich auch Unterschiede zwischen nahe verwandten Varietäten beobachten lassen: In der niederalemannischen Mundart von Basel wird für die IO-Position dieselbe Strategie verwendet wie für die OBL-Position, wogegen in der niederalemannischen Mundart von Oberrotweil für die IOPosition dieselbe Strategie verwendet wird wie für die SU- und DO-Position. Die IO-Relation scheint somit im deutschen Diasystem am wenigsten stabil zu sein.

Relativpronomen sind in den Dialekten seltener, als man aufgrund der Standardsprache vermuten würde. Das passt zu Resultaten aus der historisch-vergleichenden Linguistik und aus der Sprachtypologie: Relativpronomen kommen fast ausschließlich in europäischen (Standard-)Sprachen vor. Von den hier behandelten Formen scheint besonders welcher kein natürlicher Typ zu sein (cf. Fußnote 8). In den behandelten Varietäten treten dagegen häufiger unflektierte Partikeln (mit oder ohne weitere Elemente) auf; in den höheren Positionen ist es häufig eine unflektierte Partikel allein, in den tieferen Positionen (vor allem in der OBLPosition) meist eine Partikel in Verbindung mit einem (von einer Präposition abhängigen) Pronomen oder einem sonstigen resumptiven Element.

In einer diachronen Perspektive können aus flektierten Pronomen unflektierte Partikeln entstehen. Anhand von unflektiertem was kann zunächst die Entwicklung von einem Interrogativpronomen zu einem Relativpronomen gezeigt werden. In der weiteren Entwicklung kann sich das ursprüngliche Pronomen zu einer unflektierten Relativpartikel entwickeln (was wahrscheinlich die adäquate Analyse für viele der als unflektiertes was vorgestellten Subtypen ist), die sich dann zu einem allgemeinen Subordinator weiter entwickeln kann.

Wenn man die Standardsprache mit den hier untersuchten Varietäten vergleicht, so weist sie ein völlig atypisches Bild auf: Die Standardsprache kennt fast ausschließlich flektierte Relativpronomen; sie kennt beispielsweise praktisch gar keine relativsatzeinleitenden Partikeln oder gespaltene Pronominaladverbien, und die auftretenden Relativpronomen werden nicht mit Partikeln kombiniert. Das System, das die Standardsprache aufweist, findet sich in kei- 
nem der untersuchten Dialekte wieder; es ist darum wohl nicht auf eine natürliche Entwicklung, sondern auf Standardisierung unter dem Einfluss anderer europäischer Standardsprachen zurückzuführen. Das System der neuhochdeutschen Standardsprache hat keine direkt vergleichbare dialektale Basis und ist in diesem Sinn unnatürlich.

\section{Literaturangaben}

Albrecht, Karl (1881): Die Leipziger Mundart. Grammatik und Wörterbuch der Leipziger Volkssprache. Leipzig.

Besch, Werner et al. (eds.) (1983): Dialektologie. Ein Handbuch zur deutschen und allgemeinen Dialektforschung. Bd. 2. Berlin/New York. (= Handbücher zur Sprach- und Kommunikationswissenschaft 1.2).

Binz, Gustav (1888): Zur Syntax der baselstädtischen Mundart. Stuttgart.

Bock, Karl Nielsen (1933): Niederdeutsch auf dänischem Substrat. Studien zur Dialektgeographie Südostschleswigs. Kopenhagen/Marburg. (= Deutsche Dialektgeo-graphie 34).

Born, Walter (1978): Kleine Sprachlehre des Münsterländer Platt. Münster.

Fleischer, Jürg (2002): Die Syntax von Pronominaladverbien in den Dialekten des Deutschen. Eine Untersuchung zu Preposition Stranding und verwandten Phänomenen. Stuttgart/Wiesbaden. (= Zeitschrift für Dialektologie und Linguistik, Beihefte 123).

Fleischer, Jürg (2004a): "A typology of relative clauses in German dialects". In: Kortmann, Bernd (ed.): Dialectology meets typology. Dialect grammar from a cross-linguistic perspective. Berlin/New York: 211-243. (= Trends in linguistics studies and monographs 153).

Fleischer, Jürg (2004b): "Zur Typologie der Relativsätze in den Dialekten des Deutschen". In: Patocka, Franz/Wiesinger, Peter (eds.): Morphologie und Syntax deutscher Dialekte und historische Dialektologie des Deutschen. Beiträge zum 1. Kongress der Internationalen Gesellschaft für Dialektologie des Deutschen, Marburg/Lahn, 5.-8. März 2003. Wien: 6083.

Haag, Earl C. (1982): A Pennsylvania German reader and grammar. University Park/London. Hausenblas, Adolf (1914): Grammatik der nordwestböhmischen Mundart (Laut- und Formenlehre mit Textproben). Prag. (= Beiträge zur Kenntnis deutsch-böhmischer Mundarten 2).

Jacobs, Neil G. et al. (1994): "Yiddish". In: König, Ekkehard/van der Auwera, Johan (eds): The Germanic Languages. London/New York: 388-419.

Keenan, Edward L./Bernard Comrie (1977): "Noun phrase accessibility and universal grammar". Linguistic Inquiry 8: 63-99.

Lowenstamm, Jean (1977): "Relative clauses in Yiddish: A case for movement". Linguistic Analysis 3: 197-216.

Lumtzer, Victor (1896): "Die Leibitzer mundart. II. Formenlehre und syntaktisches". Beiträge zur Geschichte der deutschen Sprache und Literatur 21: 499-539.

Mark, Yudel (1942): "Der atributiver bayzats". Yidishe shprakh 2: 130-141.

Mark, Yudel (1978): Gramatik fun der yidisher klal-shprakh. Nyu-York. 
Meyer, Gustav F./Ulf Bichel (1983): Unsere plattdeutsche Muttersprache. Beiträge zu ihrer Geschichte und ihrem Wesen. Überarbeitet und neu herausgegeben von Ulf Bichel. St. Peter-Ording.

Noth, Harald (1993): Alemannisches Dialekthandbuch vom Kaiserstuhl und seiner Umgebung. Freiburg im Breisgau.

Patocka, Franz (2000): "Anmerkungen zum dialektalen Gebrauch attributiver Nebensätze". In: Pohl, Heinz-Dieter (ed.): Sprache und Name in Mitteleuropa. Festschrift für Maria Hornung. Wien: 303-311.

Pirk, Kurt (1928): Grammatik der Lauenburger Mundart. Ein Beitrag zur niederdeutschen Sprache in Ostpommern. Greifswald.

Reuter, Elvira (1989): Die Mundart von Horath (Hunsrück). Phonetik und Morphologie. Hamburg. (= Forum Phoneticum 45).

Schiepek, Josef (1899): Der Satzbau der Egerländer Mundart. Erster Theil. Prag. (= Beiträge zur Kenntnis deutsch-böhmischer Mundarten 1).

Schübel, Georg (1955): Die Ostfränkisch-Bambergische Mundart von Stadtsteinach im ehemaligen Fürstbistum Bamberg. Lautlehre und Beugungslehre. Giessen.

Suter, Rudolf (1992): Baseldeutsch-Grammatik. Dritte, überarbeitete Auflage. Basel. (= Grammatiken und Wörterbücher des Schweizerdeutschen in allgemeinverständlicher Darstellung 6).

Weinreich, Max (1973): Geshikhte fun der yidisher shprakh [cf. Weinreich 1980]. 4 vols. Nyu-York.

Weinreich, Max (1980): History of the Yiddish language. Translated by Shlomo Noble with the assistance of Joshua A. Fishman. Chicago/London. [Übersetzung von Weinreich 1973, 1-2.]

Weise, Oskar (1917): "Die Relativpronomina in den deutschen Mundarten". Zeitschrift für Deutsche Mundarten: 64-71.

Wiesenhann, Tjabe (1936): Einfiuhrung in das ostfriesische Niederdeutsch. Leer. [Nachdruck 1977.]

Wiesinger, Peter (1983): "Die Einteilung der deutschen Dialekte". In: Besch et al. (1983): 807-900.

Wolf, Meyer (1974): "A note on the relative pronouns vos, vemen and velkh." In: Meyer, Wolf: Contributions to a transformational grammar of Yiddish. New York: 37-47. (= Working papers in Yiddish and east European studies 4).

Zaretski, Ayzik (1921): "Tsu Sholem-Aleykhems verter seyder (ort fun perzonvort in zats)". Di yidishe shprakh 1: 5-10. 